\title{
Pilling on Wool Fibers
}

Part 2: The Effect of the Fuzz Hairs on the Pilling Tendency

\author{
By Yasushi Omura,* Kazuo Wakayama,** Tomoichi Inoue** Members, TMSJ \\ *Fuculty of Education, Gifu University, Gifu \\ **Fuculty of Textile Fibers, Kyoto University of Industrial Arts and Textile Fibers, Kyoto
}

Based on the Journal of the Textile Machinery Society of Japan, Proceedings, Vol.24 No.1/2, T1-8 (1971)

\begin{abstract}
Pilling is affected by the amount of fuzzes on the knitted fabric which are produced by abrasion. Therefore, it ought to be studied from the point of yarn structure. In this paper, we choose the number of first twists of knitted yarn as an element leading to the fuzz formation and investigate the influence on the pilling phenomena.

(1) The less the number of first twists, the more the fuzzes produced by abrasion in amount and number and the longer they in length. This tendency is conspicuous during the initial sponging cycles.

(2) The amount, length and number of fuzzes on the knitted goods are variable to have the maximum point as the function of sponging cycles. These three factors take the maximum point at a time. The less the number of first twists, the slower the time to take the maximum point.

(3) The increment $\Delta \delta$ of fuzzes on the knitted goods is shown by the equation $\Delta \delta=k^{\prime}$ $(N \cdot \Delta \bar{l}+\bar{l} \cdot \Delta N)$, where $k^{\prime}$ is constant, $\delta$ amount of fuzzes, $\bar{l}$ length of fuzzes, and $N$ number of fuzzes. And it is mainly controlled by the second term $\bar{l} \cdot \Delta N$ during the comparatively initial stage of abrasion.

(4) The variations in weight of pills on the knitted fabric show the quadratic increase against $\delta$ in the increasing zone of $\delta$, and are the linear decrease against $\delta$ in the decreasing zone of $\delta$. Moreover, the gradients of these curves are discussed through pilling phenomena.

(5) The speed $V_{W R}$ of pill formation in weight increases radically with $\bar{l}$. And the increase of $V_{W R}$ is rather more due to the increment of the unit weight $w$ of pill than the number $n_{P}$ of pills.
\end{abstract}

(6) The critical length of fuzz to form pilling on wool fiber is about $9 \mathrm{~mm}$.

KEY WORDS: PILls, PILling, WOOL, KNITTED fabrics, ABrasion, FUZZ, TWIST, PILl WEAR OFF.

\section{Introduction}

In previous paper ${ }^{[1]}$, observations were made of basic problems of pill formation, its growth and wear-off with a result that the pilling will be affected by the amount of short fibers removable (consequently the amount of fuzzes). This amount of fuzzes has to be considered (when testings are made using the testing materials made up uncer the same knitting conditions) as a yarn structure.

In present paper, we choose as the e'ement associated with fuzzes formation the number of the first twists. It is one of e'ements which constitute yarn. With the number of the first twists as a parameter, the change of fuzz conditions and its effect on pilling phenomenon in particular amount of pills, unit weight of pills and speed of pill formation in weight, are investigate 1 into in order to obtain fundamental informations by which to clarify the pilling phenomenon.

\section{Testing Material Used}

Australian wool fibers were mixed so that a predetermined averaged quality and count might te obtained, and were spun into yarn through a worsted spinning system. The testing materials used for testing is knitted fabrics made of the yarns thus made. The standard specifications of the yarn is mentioned below. For the testing with the number of the twists as a parameter, only the items to be tested are somewhat changed to make different samples. 
In this case the other items of the standard specifications are fixed.

(Standard specifications of yarns used for fabric)

(1) Count $4 / 18.5$

(2) Number of twists First twist (Z) $250 \mathrm{tpm}$

Final twist (S) $\quad 160 \mathrm{tpm}$

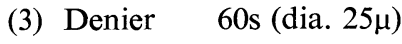

(4) Moisture regain $15 \%$

(5) Oil content after scouring $\quad 0.462 \%$

The knitting method with the above specified yarns is fixed as follows: A circular rib fabric is produced by a weft knitting machine. The number of stitches is 35 in wale and 42 in course per $10 \mathrm{~cm}$. This testing material was left at a place under the standard conditions $\left(20^{\circ} \mathrm{C}, 65 \% \mathrm{RH}\right)$ more than 24 hrs.

\section{Experimental Method}

\section{3-1. Pilling Tester}

We use a Brush \& Sponge Pilling Tester. The specifications of the tester is the same as in the previous article ${ }^{[1]}$. In the ordinary pilling testing, it was customary to sponge the material after brushing. However, in this testing, to show up the difference in pilling between materials, we sponge the materials without brushing.

3-2. Measurement of Amount of Pills and Amount of Fuzzes

(1) Measurement of amount of pills

We assess the pilling as a weight as in the previous article [1]. That is to say, after a required pilling test, we cut out the pills sticking on the knitted fabric with a pair of thin-bladed surgical scissors, and piled and weighed them by means of a precision scale. The amount of pills was measured per area of $500 \mathrm{~cm}^{2}$ of knitted fabrics and was converted, as shown in figure, into amount of pills per $\mathrm{cm}^{2}$.

The method of assessment by counting the pills sticking on the knitted goods was also used in our test, the pills per in $^{2}$ of knitted fabrics being counted and converted, as shown in the figure, into values per $\mathrm{cm}^{2}$.

(2) Measurement of unit weight $w$ of pills

To produce pills on the knitted fabric the testing material was abraded for a given time (cycles). We selected the largest 40 pills out of the pills thus produced on the material of $100 \mathrm{~cm}^{2}$. We weighed them and put their averaged value into the unit weight $w$ of pills.

(3) Measurement of amount of fuzzes

We used a singeing method as in the previous article ${ }^{[1]}$. Namely, put the weights before and after singeing into $W_{A}$ $\left(\mathrm{g} / \mathrm{cm}^{2}\right)$ and $W_{B}\left(\mathrm{~g} / \mathrm{cm}^{2}\right)$ and the amount $\delta\left(\mathrm{g} / \mathrm{cm}^{2}\right)$ of fuzzes sticking on the surface of the knitted fabric is given by

$$
\delta=W_{A}-W_{B}
$$

Besides, put the amount of the pills worn off into $W_{O}$ $\left(\mathrm{g} / \mathrm{cm}^{2}\right)$, and the total forming amount of pills $m_{f}\left(\mathrm{~g} / \mathrm{cm}^{2}\right)$ is given by the following equation:

$$
m_{f}=\delta+W_{o}=\left(W_{A}-W_{B}\right)+W_{o}
$$

where $W_{A}, W_{B}$ and $W_{o}$ are shown by converting the measured value for an area of a knitted fabric of $500 \mathrm{~cm}^{2}$ into the value for $\mathrm{cm}^{2}$. The measurement of weight was made under an absolutely dry condition, which was then converted to obtain a moisture regain of $15 \%$.

\section{3-3. Measurement of Fuzz Length}

We cut out fuzzes nearest the surface of the yarn, arranged them on the slide glass dropped with ceder oil, and measured them by means of microscopy.

3-4. Continuous Measurement of Stuck Pill and Fuzz Amounts

The amounts of pills and fuzzes worn off out of the knitted fabric are obtainable with one material through the timepassage data. However, since the amount of pills sticking on the knitted fabric, the amount of fuzzes and their lengths are varied by times of abrasion, the pills and fuzzes have to be cut out of the knitted fabric after every abrasion. And so one material cannot be used for continuous testings (cycles).

Accordingly, we made many samples out of the same lot. We whacked up them for respective testings to obtain data by every abrasion. Therefore, in this case, each of data protted against one curve is datum based on various samples made up under the same conditions.

However, as mentioned in the previous article ${ }^{[1]}$, the stuck pill weight curve obtained as a series of data based on various samples made up under the same conditions is comparable with the worn-off pill weight curve obtainable with one material.

\section{Experimental Results and Discussion}

It was already known in the previous article ${ }^{[1]}$ that the fuzzes taken place by abrasion has a great effect on the pilling phenomenon. In this article, we take as a parameter the final twists of the yarn which constitutes the knitted fabric. The effect of change of the first twists on forming fuzzes and also of change of this fuzzes on pilling phenomenon are observed.

\section{4-1. Effect of Change of First Twists on Forming Fuzzes}

\section{4-1-1. Slippage resistance of fibers}

Hardness or ease of fuzz formation by abrasion on the yarn is governed by the slippage resistance of fibers from the yarn. The factors associated with slippage resistance of fibers are twisting conditions and fiber properties (Young's modulus, coefficiency of friction, elongation, breakage strength, fiber length, denier, crimp shape, etc.). Here, we deal with the yarn made of the same material and the properties of above-mentioned fiber can be considered fixed.

Fig.1 shows breakage strength, Young's modulus and elongation of woolen single yarn $(1 / 18.5)$ when only the number of twists are varied.

The more the number of twists of the yarn made of the same fibers, the higher the strength and Young's modulus, and the less the elongation. Accordingly Fig.1 shows re- 


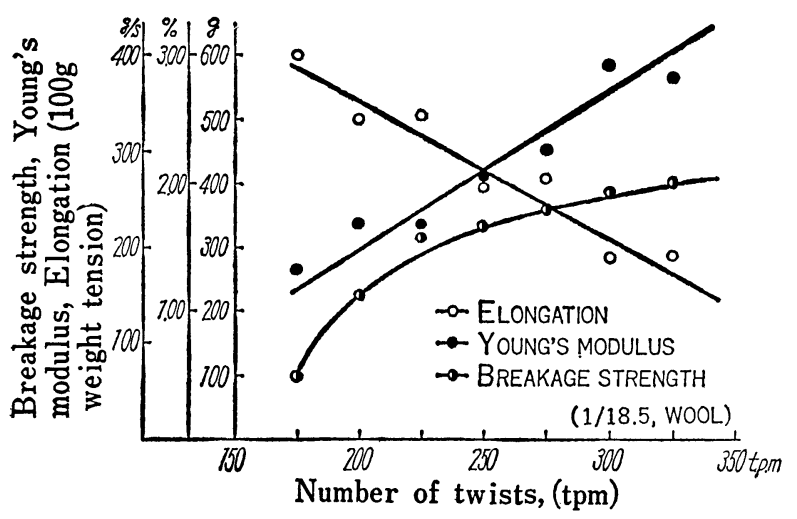

Fig. 1 Variations in elongation, Young's modulus and breakage strength as the sequel to the number of twists of a woolen single yarn

alistically that the more the number of the first twists the greater the slippage resistance of fibers out of the yarn. 4-1-2. Total amount $m_{f}$ of fuzzes, amount $\delta$ of stuck fuzzes, fuzz length $\bar{l}$ and number $N$ of fuzzes

Using the samples with varied number of first twists, the relation between the abrasion cycles and the total amount of eventual fuzzes $m_{f}$ is shown in Fig.2. Fig.3 shows the relation between the abrasion cycles and the amount $\delta$ of fuzzes stuck on the knitted fabric, using the same samples as in Fig.2.

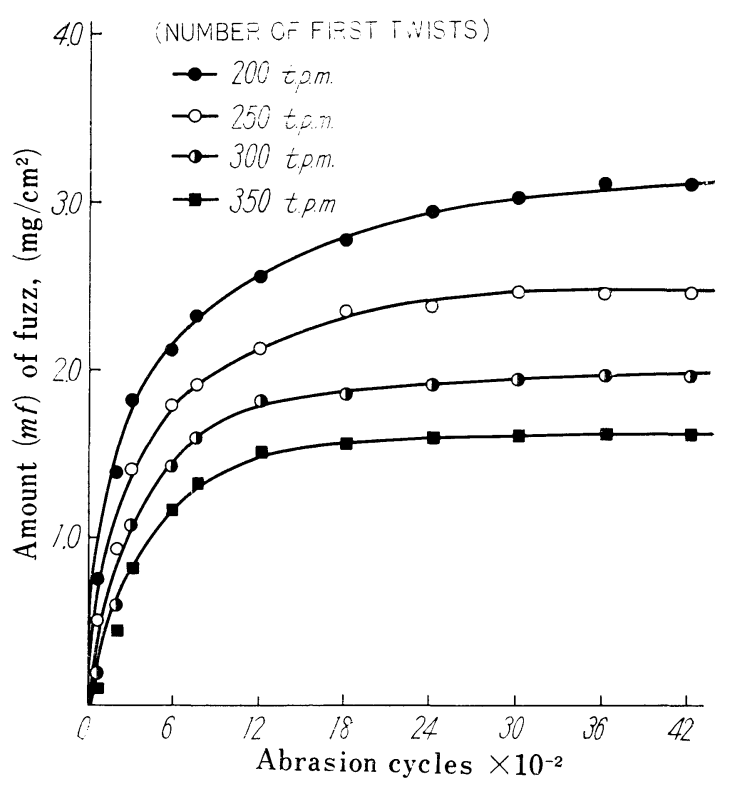

(4/18.5, final twist $160 \mathrm{tpm})$

(Data based on various samples made up under the same conditions)

Fig. 2 Relation between abrasion cycles and total amount $\left(m_{f}\right)$ of füzz (including stuck and worn-off fuzzes), using the samples with varied number of first twists

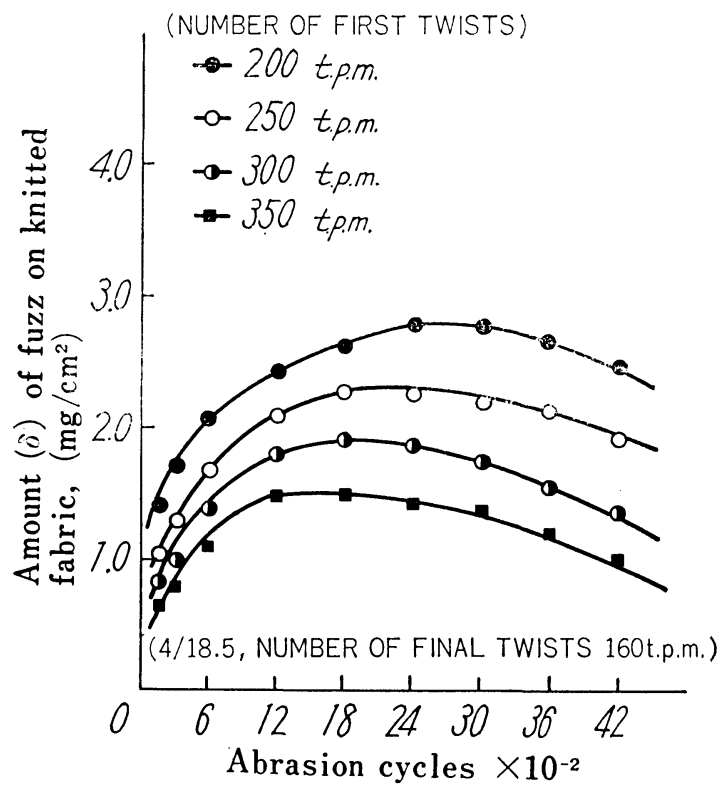

(Data based on various samples made up under the same conditions)

Fig. 3 Relation between abrasion cycles and amount $(\delta)$ of fuzz on knitted fabric

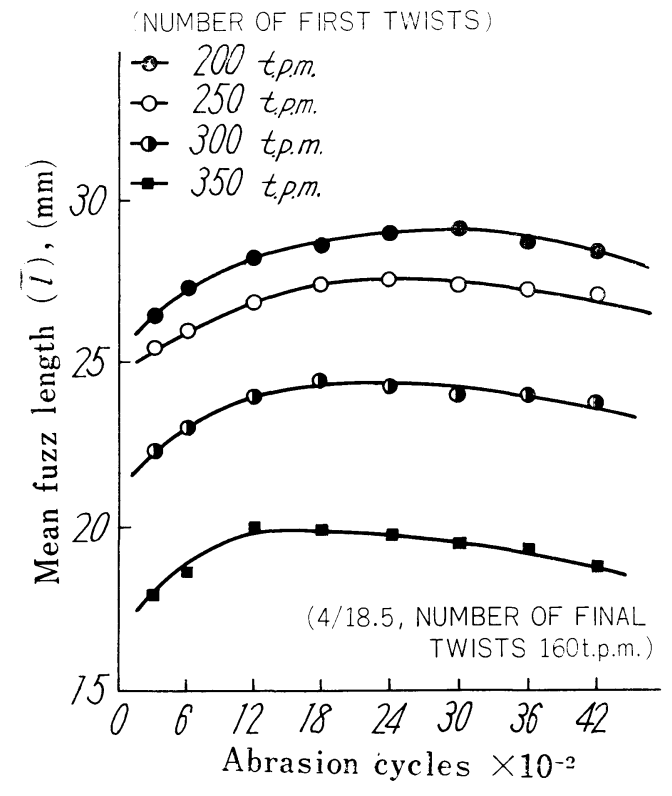

(Data based on various samples made up under the same conditions)

Fig. 4 Relation between abrasion cycles and mean fuzz length $(\bar{l})$

Fig. 4 shows the mean fuzz length $\bar{l}$ at every abrasion of respective samples. A histogram of fuzz length $l$ after 600 abrasions is shown in Fig.5. 


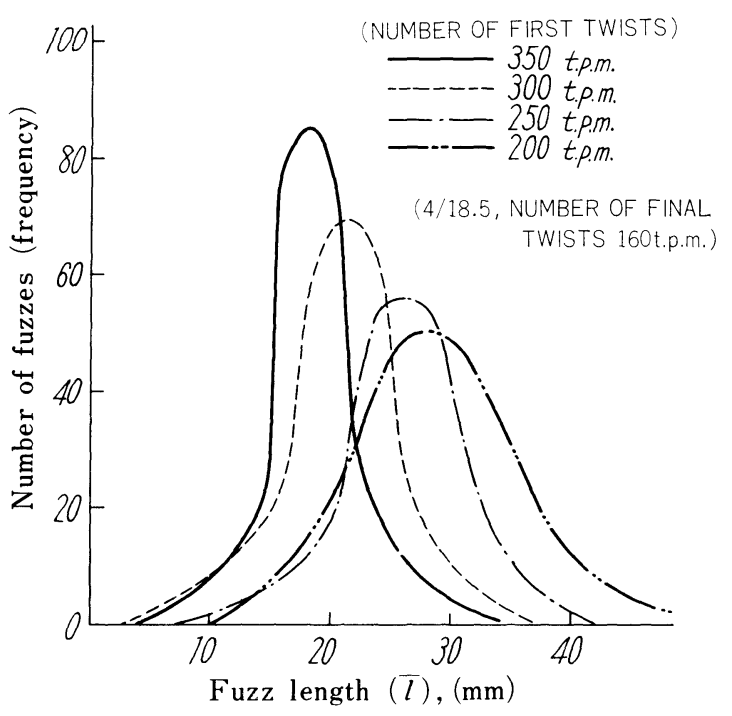

Fig. 5 A histogram of fuzz length $l$ after 600 abrasions

Besides, a time-passage of the number $N$ of fuzzes by abrasion is shown in Fig.6. The number of fuzzes when the fuzz length was measured cannot te fixed tecause a bit of the area of samp'e enployed is repeatedly se'ested at random. The number of fuzzes re: given area of sample to render the number of fuzzes may be obtained by expanding the concept of mexn fuzz length $\bar{l}$. Now consider the total $\operatorname{sum} 4 \delta / \pi d^{2} \rho$ of all fuzz lengths per unit area $(\hat{o}$ is amount of fuzzes per unit area, ", is fiber density, $d$ is fiber diameter). This devision by the total number of fuzzes per unit area $N$, gives mean fuzz length $\bar{l}$.

Conseguently,

$$
\mathrm{N}=\frac{4}{\pi d^{2} \rho} \cdot \frac{\delta}{\bar{l}}=k \frac{\delta}{\bar{l}}
$$

where $k$ is a constant determined by the sample fiber. When the samples used for experiment were measured, $d=65 \mu$ and $\rho=1.30 \mathrm{~g} / \mathrm{cm}^{3}$. Therefore, $k=4-d^{2} \rho=$ $23.19 \mathrm{~cm} / \mathrm{mg}$. The amount $\delta$ of fuzzes sticking on the surface of the knitted fabric after predetermined abrasions is known from Fig.3, and $\bar{l}$ at this time is known from Fig.4. If these values are substituted into eq.(1), the timepassage of number $N$ of fuzzes can te calculated. The number of fuzzes shown in Fig.6 is what was obtained by such calculations. It see.ns impossible to obtain such results by meas:Iring the number of fuzzes.

Figs. 2 to 6 can te explained together.

The amount $m_{f}$ of fuzzes produced by abrasion is very marked at the beginning, then the rate of fuzz formation begins to decrease and takes a stationary valuc. This propensity is true of every sample. The less the numter of first twists, the more the amount of forming fuzzes, and the later the time reaching the stationary value.

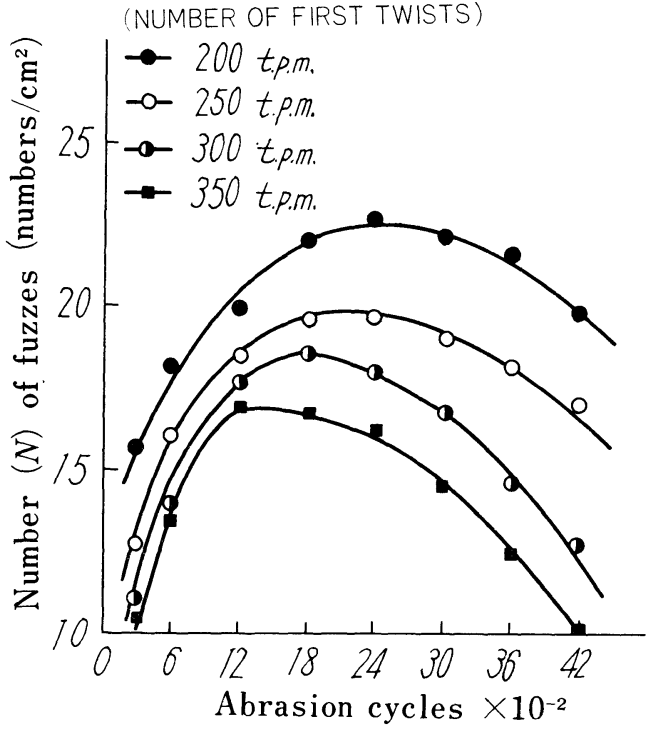

(Data based on various samples made up under the same conditions) (4/18.5, final twist 160 tpm)

Fig. 6 Relation between abrasion cycles and number (N) of fuzzes

On the other hand, the amount of fuzzes sticking on the surface of knitted fabric has a time point taking a maximum value against the abrasion times. Since this time point, the amount of fuzzes sticking on the surface of the knitted fabric tegins to decrease and the wear-off of fuzzes becomes conspicuous. This means that the amount of wear-off of fuzzes excceds the amount of forming fuzzes. On this time point, the less the numter of the first twists, the later this time-point comes, and the fuzzes will not ke worn off easily from the surface of the knited fabric.

Then, let us consicer the length of fuzzes produced. The mean fuzz length $\bar{l}$ varies with the abrasion times and takes a maximum valce. The less the number of first twists, the later the maximum value of $\bar{l}$ comes. The fuzz lengths are presented as their average value $\bar{l}$ in Fig.4. The variation status of fuzz 'ength $/$ can be recognized from Fig.5. By and large, the more the number of twists, the steeper the curve, and the nearer the curve itself toward the shorter fuzz length. This is presumably because the more the numter of twists, the more uniform and the shorter fuzz lengths.

The number $N$ of fuzzes can ke known from Fig.6. As for respective samples, the number $N$ of fuzzes is apt to increase until the times of abrasion are increased upto a certain numter, since which they will decrease. This decrease is presumably kecause the fuzzes were worn off because of fiter slippage and wear-out. The time point (cycles) when the number $N$ of fuzzes takes the maximum is that the less the number of the first twists the later it is. Resides, in the case of less number of the first twists, the number $N$ of fuzzes is apt to increase. This degree will te 
that the number $N$ of fuzzes is increased by 6 to $7 \%$ on the average if the number of first twists is decreased by a cegree of $50 \mathrm{tpm}$. Also, the increase of the number $N$ of fuzzes with change of number of the first twists can te understood from the slopes of the respective curves where the less the number of twists the larger the increase.

From Figs.3, 4 and 6 it is known that at the time point when the amount $\delta$ of fuzzes sticking on the surface of knitted fabric takes a maximum point (abrasion cycles), both the fuzz length $\bar{l}$ and number $N$ of fuzzes of respective samples show a maximum value. Accordingly, at the time point when $\delta, \bar{l}$ and $N$ show maximum point, the fuzzes on the knitted fabric are apt to be entangled with each other due to abrasion, and be major causes for pilling phenomenon.

4-1-3. Relation among fuzz length, number of fuzzes and amount of fuzzes

It is known from the above observation that the amount $\delta$ of fuzzes, fuzz length $\bar{l}$ and the number $N$ of fuzzes are varied by the times of abrasion, and that they take their maximun values at the same time point. The problem here may be how the contribution of fuzz length $\bar{l}$ and the number $N$ of fuzzes to the derrease or increase of the amount $\delta$ of fuzzes is. Now, from eq.(1) put $k^{\prime}=1 / k$, then

$$
\grave{o}=k^{\prime} \bar{l} N
$$

The re'ation among the variations of $\bar{l}, N, o$ within $J T$ is given by

$$
\lrcorner \bar{o}=k^{\prime}(\bar{l} \cdot\lrcorner N+N \cdot\right\lrcorner \bar{l}\right)
$$

In the eq.(3), the first and second terms at the right sice show the contribution of the numter $N$ of fuzzes and fuzz length $\bar{l}$ to the variation $\lrcorner j$ of the amount of fuzzes due to the change of abrasion cyc'es. $J^{\prime}$ and $J N$ can te calculated as a variation due to a certain number of atrasion from the gradient of tangent line of respective curves which show the variation of fuzz length $\bar{l}$ and number $N$ of fuzzes at tinces $n$ of abrasion in Figs. 4 and 6. Fig. 7 shows the relation between the abrasion cycles and each terms at the right side of ex.(3) in the case where the number of first twists is $250 \mathrm{tpm}$ and $300 \mathrm{tpm}$. The first term $\bar{l} \cdot\lrcorner N$ shows remarked variation with the number of first twists. The second term $N \cdot \Delta l$ shows an extremely little variation. Particularly at the initial stage of abrasion, the first term $l \cdot \Delta N$ shows extremely large value. Namely, it is known that the variation $\lrcorner \grave{o}$ of amount of fuzzes is more affected by the variation $\bar{l} \cdot J N$ of number of fuzzes than by the variation $N \cdot \Delta \bar{l}$ of fuzz length at the initial stage of abrasion.

4-2. The Effect of Fuss Status on Pilling Phenomenon

In the item 4-1, it is known that the amount $\delta$ of fuzzes, fuzz length $\bar{l}$ and the number $N$ of fuzzes are varied by abrasion times. Here we discuss how the pilling phenomenon will be affected by the variation of these fuzzes. 4-2-1. Effect of fuzz amount $\delta$ on pilling phenomenon

The variation of pilling amount sticking on the surface

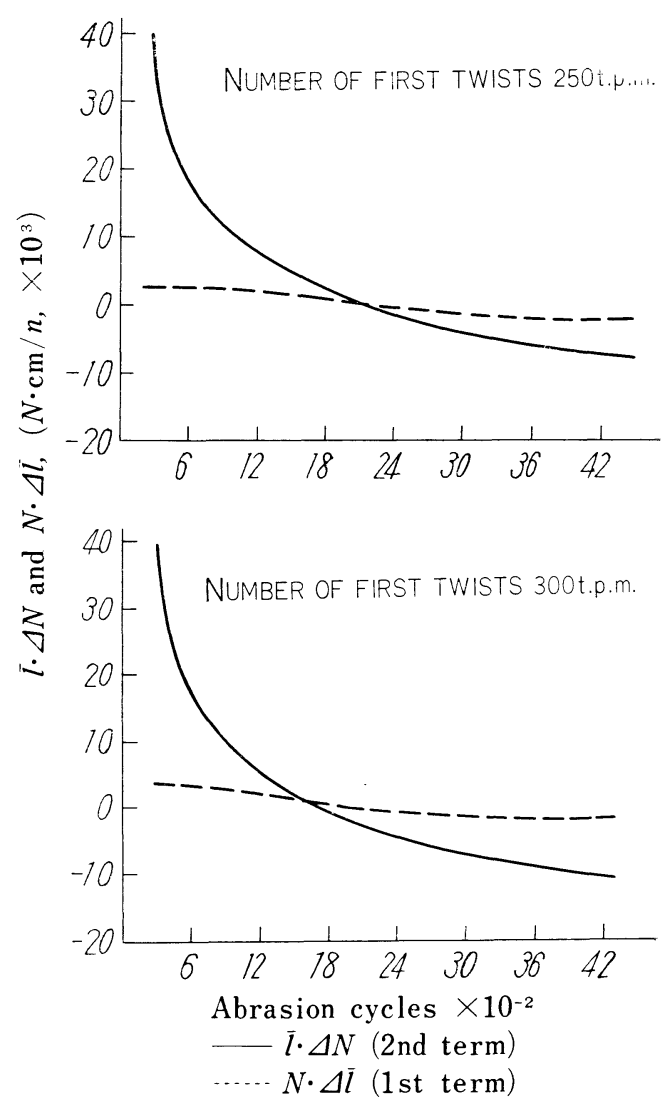

Fig. 7 Relation between abrasion cycles and each terms at the right side of eq.(3)

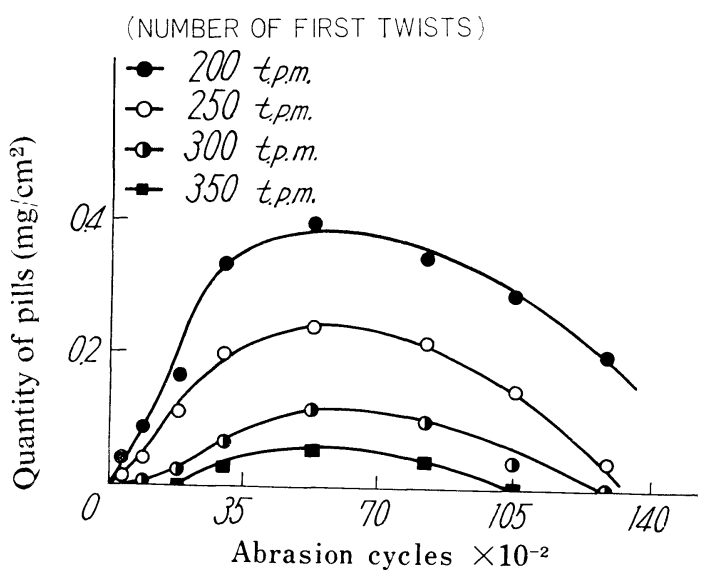

(Data based on various samples made up under the same condition)

Fig. 8 Variations in pilling amount sticking on the knitted fabric by abrasion cycles

of the knitted fabric by respeztive cyc'es of abrasion is shown in Fig.8. Figs. 9 and 10 show the re!ation tetween the amount $\delta$ of fuzzes taking the abrasion cycles as a parameter and the amount of pills sticking on the surface of the 


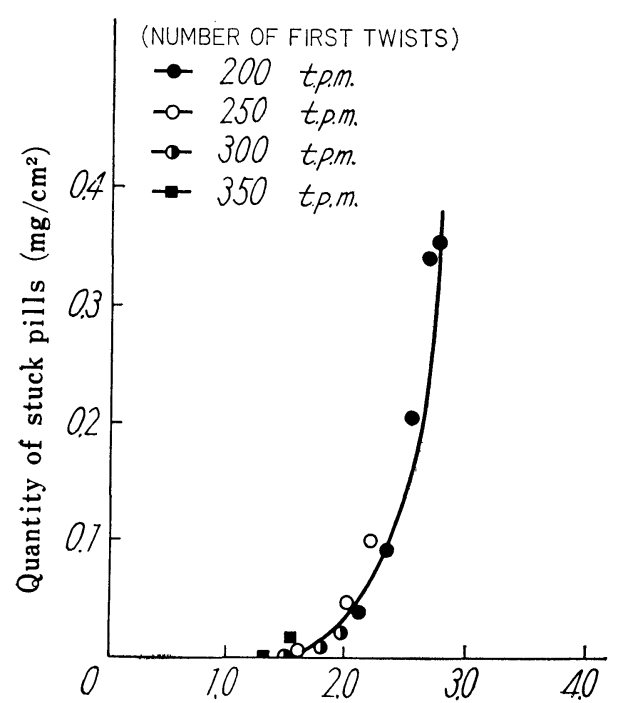

Amount $(\delta)$ of fnzz on knitted fabric $\left(\mathrm{mg} / \mathrm{cm}^{2}\right)$

Fig. 9 Relation between amount $(\delta)$ of fuzz and quantity of pills on knitted fabric, in the increase region of $\delta$

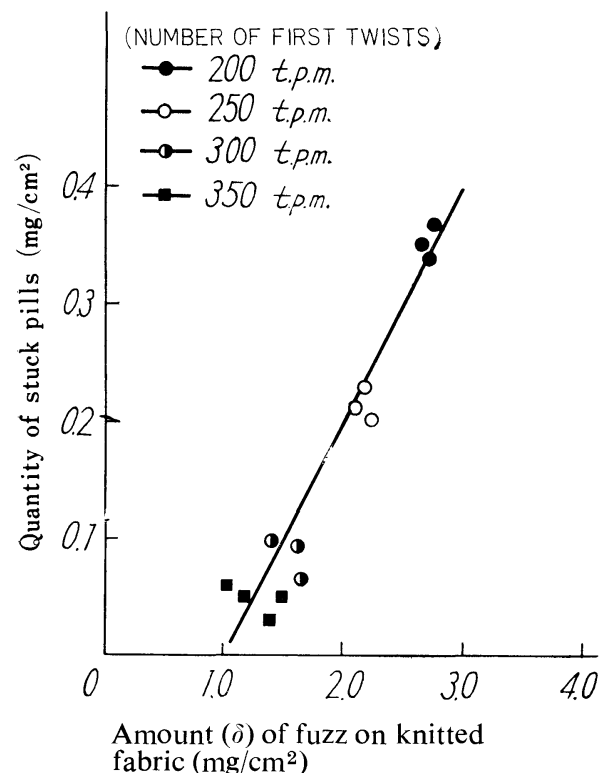

Fig. 10 Relation between amount $(\delta)$ of fuzz and quantity of pills on knitted fabric, in the decrease region of $\delta$

knitted fabric, which are derived based on Figs. 3 and 8. They are grouped in respective regions of increase and decrease of fuzzes. The reason is that even if there is the same amount of fuzzes on the knitted fabric there may be a difference in pilling phenomenon between in the case of $d \delta / d t>0,(t:$ time of abrasion) and in the case of $d \delta / d t<0$.

From the figure, in the increasing region of the amount $\delta$ of fuzzes, the variation in amount of stuck pills shows the quadratic increase against $\delta$. Since this tendency comes over this curve regardless of the number of the first twists, the amount of stuck pills, if the material is the same, will come to be a problem as only one element of $\delta$. From this, it is known that the amount of stuck pills will be affected by the slight degree of increase or decrease of $\delta$, and that by removing the fuzzes sticking on the knitted fabric at the initial stage of abrasion said in 3-4 of previous article ${ }^{[1]}$ (after 300 times of sponging), the eventual amount of pills after then will be extremely reduced.

In the decrease region of the amount $\delta$ of fuzzes, it is difficult to recognize the propensity only from one sample with fixed number of first twists. Judging collectively from data obtained from the samples with different numbers of the first twists, it is clear that the relation between the amount of stuck pills and $\delta$ will be linear.

Besides, at the both regions of increase and decrease of the amount of fuzzes, the degree of the increase and decrease of the amount of stuck pills will be different and the gradient which shows the decrease of stuck pill amount will be more gradual than that of the increase (except the beginning portion of the increase region of $\delta$ ).

The above-mentioned phenomenon is considered as follows. As mentioned in 4-1, in the increase region of the amount $\delta$ of fuzzes, the number $N$ of fuzzes and fuzz length $\vec{l}$ will increase and the fibers bristling from the surface of the knitted fabric are apt to be entangled with one another. What is more, since the time points which $N$ and $\bar{l}$ show the maximum values areagreed, this entangling propensity will be further facilitated as they near this point, and the marked increase of the amount of pills will be seen. From the above reasons, in this region, the amount of stuck pills does not show a linear relation to the amount $\delta$ of fuzzes but a quadratic variation. In the decrease region of the amount of fuzzes, as mentioned in the previous article $^{[1]}$, the formation of fuzzes will be less and the fuzz length be comparatively short. Besides, since the fuzzes is reduced in strength due to abrasion damage, the formation of pills will te small and the speed of the wear-off of pills will be higher. And what is more, $d W_{o} / d t=d n_{o} / d t \cdot w=$ const. ( $W_{o}$ : integral values of weight of wear-off of pills, $n_{o}$ : the number of worn-off pills, and $w$ : unit weight of pills) is recognized to be correct except the beginning portion of abrasion. ${ }^{[1]}$ From these, in the region where the amount of fuzzes is decreasing, there is very few pill formation and its growth, while since the amount of pills on the knitted fabric wears off at a constant rate the amount of pills decreases linearly against the abrasion. It is known from Fig. 8 that in the decrease region, as the abrasions are increased, the amount of stuck pills will decrease much more linearly. On the other hand, in the decrease region of $\delta$ in Fig. 3 the amount $\delta$ of fuzzes also decreases nearly 
linearly to abrasion, so that in this region the amount of stuck pills and $\delta$ show a linear relation.

From these, the amount of stuck pills in the decrease region of the amount $\delta$ of fuzzes and $\delta$ show the linear relation whose linear gradient is varied by the abrasion strength of fibers related to the wear-off speed of pills in weight $\left(d W_{o} / d t\right)$.

4-2-2. Fuzz length and pilling phenomenon

In the observation of fuzz length and pilling phenomenon, it is practically difficult to discuss separately the effect of fuzz length and effect of the number of fuzzes.

Fuzz length and fuzz number can be grasped as a separated data, but pilling itself cannot be grasped as data when fuzz length and the number of fuzzes are varied both at once. However, from the practical view point of pilling when getting on the clothing, it has some meaning as it is even if they are not completely separated.

Now let us consider the effect of the fuzz length $\bar{l}$ on pill formation (actually, though the number $N$ of fuzzes is one of factors affecting the hardness or ease of pill formation, and since $\bar{l}$ and $N$, as shown in Figs. 4 and 6, show both the same propensity against the cycles of abrasion, it will be enough to observe either of them. And what is more, to reduce the amount of fuzzes, $\bar{l}$ is preferable to $N$. For the every sample with the number of twists of 200 to $350 \mathrm{tpm}$, the amount of pills and $\bar{l}$ showed a higher correlation of 0.986 to 0.988 . These are the reasons why the fuzz length is selected).

Fig.11 shows the relation between the cycles of abrasion and the speed $V_{W R}$ of pill formation in weight. The relation between fuzz length $\bar{l}$ and the speed $V_{W R}$ of pill formation in weight calculated from Figs. 4 and 11 is plotted, in Fig. 12 , for every sample at every abrasion.

From this the effect of $\bar{l}$ on $V_{W R}$ is large. The longer the $\bar{l}$ the more $V_{W R}$ increases radically. Accordingly, the pill formation rapidly proceeds with the increase of $\bar{l}$.

Here, the causes for the increase of $V_{W R}$ may be the increase of unit weight $w$ of pill and number $n_{P}$ of pills both. Fig. 13 shows the variation of $n_{P}$ by abrasion cycles. From Figs. 4 and 13, almost all samples which have the same twist of yarn take a constant value in the region where fuzz length varies. So that the increase of $V_{W R}$ does not arise from increase of $n_{P}$ but the increase of $w$. From the above, it is known easily that the longer the $\bar{l}$ the larger the $w$. Fig. 14 shows the variation of $w$ by abrasion cycles. Fig. 15 shows the relation ketween $\bar{l}$ and $w$ which were calculated from Figs. 4 and 14, which clearly shows the longer $\bar{l}$ the larger $w$.

Fig. 12 has another meaning that the pills do not occur if $\bar{l}$ is less than a certain value, i.e., $V_{W R}=0$, since the each curve passes the original point. If we seek a limit value by expanding each curve, each curve crosses at $\bar{l}=9 \mathrm{~mm}$ for every sample. In this case, the value of $\bar{l}$ has the same

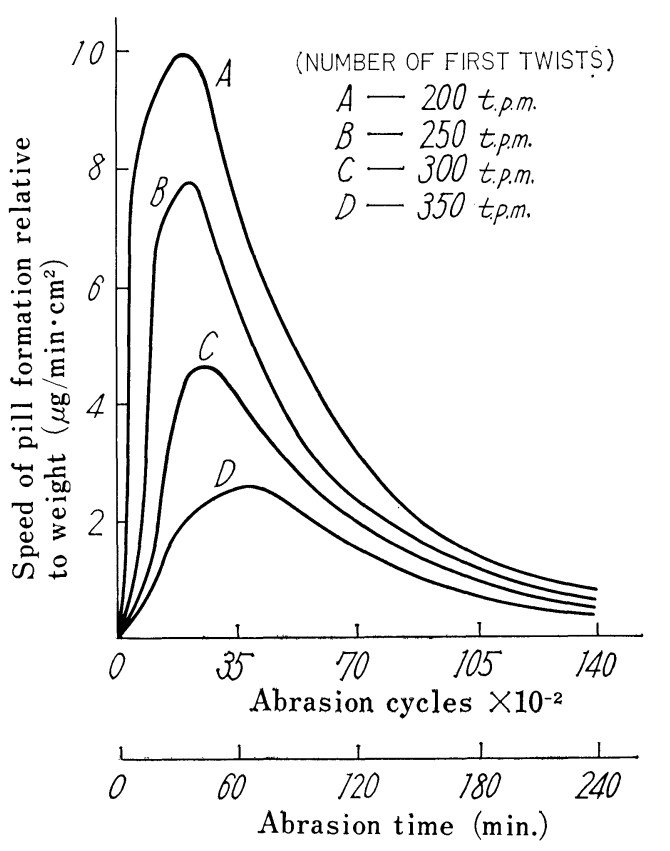

(4/18.5, final twist $160 \mathrm{tpm})$

Fig. 11 Relation between abrasion cycles and the speed $\left(V_{W R}\right)$ of pill formation in weight

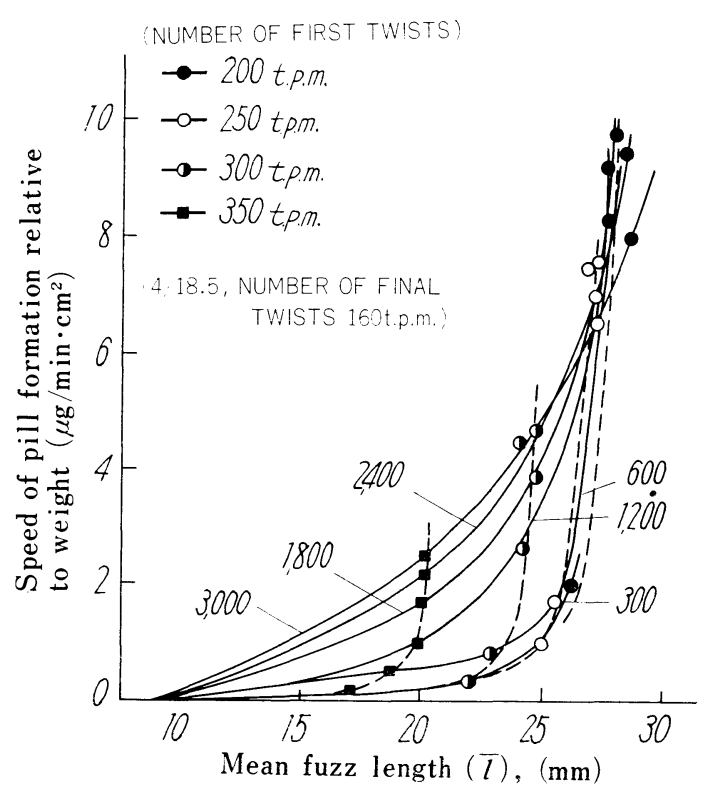

(Numbers in the figure show the number of abrasion cycles) (A solid line shows the variation of $\left(V_{W R}\right)$ by abrasion cycles and dotted line shows the variation of $\left(V_{W R}\right)$ by number of first twists)

Fig. 12 Relation between mean fuzz length $(\bar{l})$ and the speed $\left(V_{W R}\right)$ of pill formation in weight 


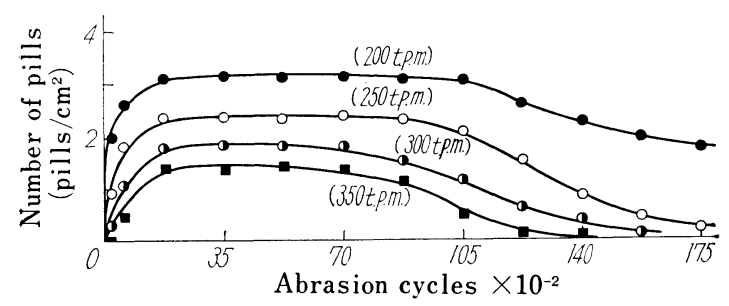

(4/18.5, final twist $160 \mathrm{tpm})$

(Numbers in parentheses show the number of first twists of the yarn which constitutes the knitted fabric.)

Fig. 13 Number of pills on knitted fabric

meaning as the critical length informed by D. Gints and E. J. Mead ${ }^{[2]}$ and agrees with their experimental value (in case of wool, 10/32 inch $=8 \mathrm{~mm}$ )

\section{Conclusion}

(1) The less the number of first twists, the greater the fuzz produced by abrasion in amount, number and length. In particular, the increase of the amount of fuzzes is conspicuous at the initial stage of abrasion. The less the number of first twists the more distinguisted the propensity.

(2) The amount, length and number of fuzzes on the knitted fabric are variable to have a maximum value against the times of abrasion, and the time point when they take the maximum value comes at a time. The time point is that the less the number of first twists the later it is, and the fuzzes do not easily worn off out of the knitted fabric.

(3) At the initial stage of abrasion, the degree $J i$ of increment is more effected by a contribution $\bar{l} \cdot \Delta N$ by the number of fuzzes than $N \cdot \bar{l}$. by fuzz length.

(4) The amount of fuzzes sticking on the knitted fabric will differ between in the increase region of the amount of fuzzes and in the decrease region. In the increase region of $\delta$, the amount of pills sticking on knitted fabric shows a quadratic variation against $\delta$, whereas in the decrease region, it decreases linearly with the cecrease of $\delta$. The gradient where the amount of stuck pills decrease is more lenient than that where it increases (except the initial portion of the increase region). In the linear relation between the amount of stuck pills in the decrease region of $\delta$ and $\delta$, the gradients of these curves differ according to the fiber abrasion strength associated with the sreed of wearoff of pills in weight.

(5) The effect of fiber length $\bar{l}$ on the speed $V_{W R}$ of pill formation in weight is larger. $V_{W_{R}}$ increases radically with $\bar{l}$. In this case, the incrcase of $V_{W R}$ is more affected by unit pilling weight $w$ than by the numter of $n_{P}$ pills.

(6) In the case of wool, the critical length of fuzz to form pilling is about $9 \mathrm{~mm}$.

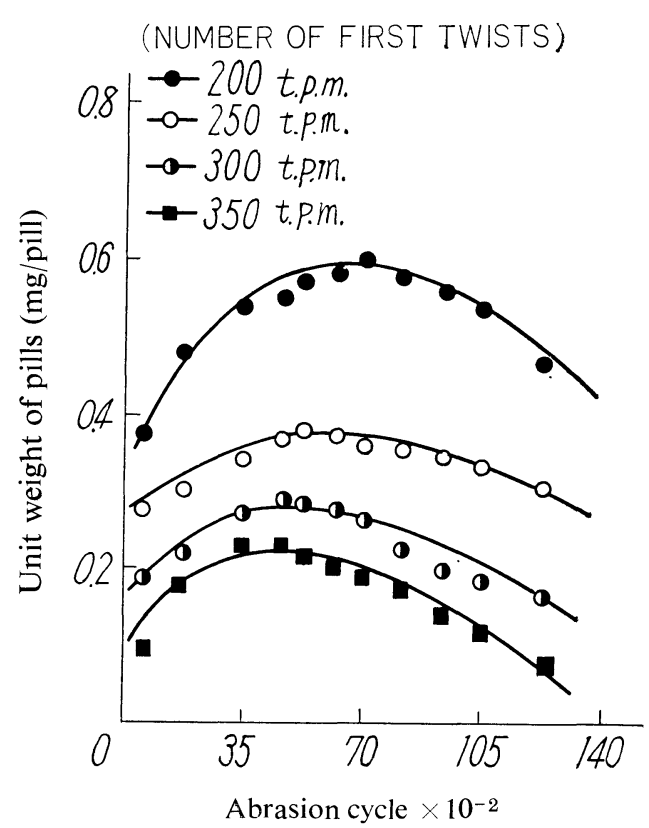

(4/18.5, final twist $160 \mathrm{tpm})$

(Data based on various samples made up under the same condition)

Fig. 14 Variation of unit weight ( $w$ ) of pills by abrasicn cycles

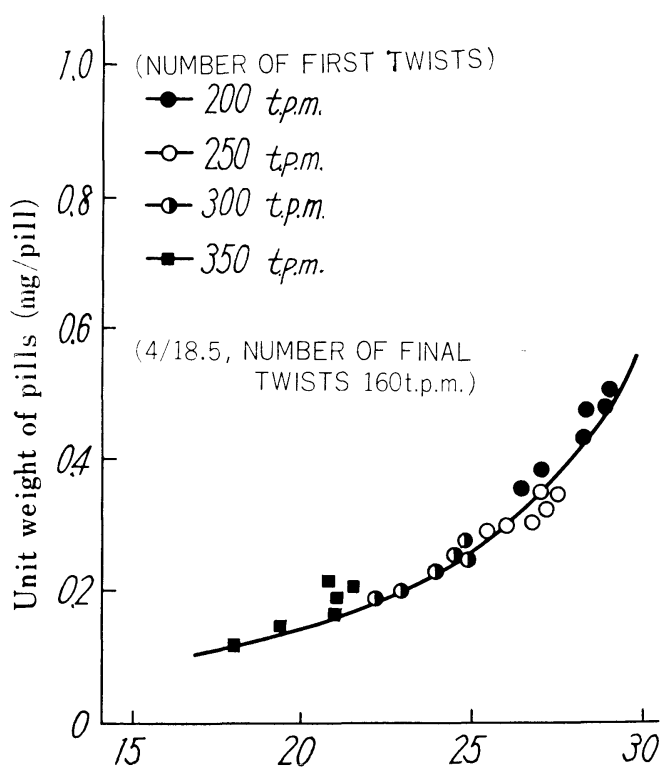

Mean fuzz length $(\bar{l}),(\mathrm{mm})$

Fig. 15 Relation between mean fuzz length $(\bar{l})$ and unit weight $(w)$ of pills

\section{Literature cited}

[1] Omura, Wakayama, Inoue; J. Text. Mach. Soc. Japan Vol.15, No.2, T45-53 (April, 1969)

[2] D. Gints, E. J. Mead; Text. Inst., 38, T151 (1947-3) 